\title{
Achievement of Developmental Milestones in Children with Idiopathic Clubfoot Treated with Ponseti Method in Lagos Nigeria
}

\author{
Famude S.O., ${ }^{1}$ Adewole O.A., ${ }^{2}$ Komolafe O.A. ${ }^{1}$ \\ ${ }^{1}$ Department of Physiotherapy, Lagos State University Teaching Hospital Ikeja, Lagos, Nigeria \\ ${ }^{2}$ Orthopaedic Unit, Department of Surgery, Lagos State University College of Medicine, Ikeja, Lagos, Nigeria \\ Correspondence \\ Famude Samuel Olawale, Lagos State University Teaching Hospital, 1-5 Oba Akinjobi Road, Ikeja, Lagos, \\ Nigeria.・Email-olawalefamude@yahoo.com
}

\begin{abstract}
SUMMARY
Clubfoot, also known as congenital talipes equinovarus (CTEV), is a congenital deformity involving one foot or both. It is a relatively common birth defect occurring in about one in every 1,000 live births. No study has been carried out to determine if there is delay in gross motor milestones in children with idiopathic clubfoot treated with Ponseti method in this part of the world. This study was conducted to investigate this.

In this prospective study, 48 children with idiopathic clubfoot who were compliant with the foot abduction brace were enrolled. Parents were interviewed repeatedly to obtain the ages of achievement of 8 motor milestones and the data was stored and analysed using SPSS version 17.

Forty-eight children with mean age of 1.2 months as at first casting and a male: female ratio of 3.8:1 satisfied the inclusion criteria. Of the eight milestones studied, the mean age of achievement of one of them was significantly greater $(\mathrm{P}<0.05)$ compared with normative data. Pre-ambulatory and crawling milestones showed no significant difference, but independent ambulation was achieved up to 2 months later.

Although there were significant delays in upright ambulation in children with idiopathic clubfoot treated with Ponseti method, they all walked.

These findings should enable clinicians alleviate the concerns and fears of the parents of children with idiopathic clubfoot and assure them of a positive prognosis regarding developmental milestone achievement in spite of delays.
\end{abstract}

KEY WORDS: Congenital talipes equinovarus, idiopathic clubfoot, Ponseti method, developmental milestones

\section{INTRODUCTION}

A club foot or clubfoot, also known as congenital talipes equinovarus (CTEV), is a congenital deformity involving one foot or both (Ponseti, 2000). The affected foot appears to have been rotated internally at the ankle, with fore foot adduction, heel varus, equinus and a medial cavus (Staheli, 2009). CTEV may be classified as idiopathic, syndromic or neurological. In the idiopathic variety, there are no other congenital anomalies. In the syndromic type, there are associated anomalies such as arthrogryposis multiplex congenita and the neurological clubfoot is associated with neurological deficits as in meningomyelocele (Lynn, 2009). Without treatment people with club feet appear to walk on their ankles or on the sides of their feet, however with treatment, most patients recover completely during early childhood and are able to walk and participate in athletics as well as children born without CTEV (Lynn, 2009).

Clubfoot is a relatively common birth defect occurring in about one in every 1,000 live births, and approximately half of the cases are bilateral, it occurs in males twice as 
frequently as in females (Dobbs and Gurnett, 2014). Scholars in Nigeria have reported that it is the commonest orthopaedic anomaly (Adewole et al, 2009). Over the past decade, Ponseti management of CTEV has become accepted throughout the world as the most effective and least expensive, with a success rate exceeding $95 \%$ (Adewole et al, 2014). The treatment is started soon after birth and this is done by weekly serial manipulation and casting in maximum correction to correct the various deformities (Omololu et al, 2005). A percutaneous tenotomy is performed where necessary to correct the equinus deformity and the foot is placed in a last cast for 3 weeks (Morquende et al, 2004). The foot correction is maintained by using a foot abduction brace worn 23 hours a day for the first 3 months after cast removal and then at nap and night times until approximately $3-4$ years of age (Ponseti, 1963), as non-compliance with the brace is the commonest reason for reoccurrence (Dahaung at al, 2014). Early experience of this treatment in Nigeria has revealed results similar to other parts of the world (Adewole et al, 2014).

This study was undertaken to compare gross motor development in children with CTEV who have undergone treatment using the Ponseti method with normative developmental data.

\section{DEFINITION OF TERMS}

Congenital talipes equinovarus (Clubfoot) - is a developmental deformation of the foot (Lynn, 2009) in which the foot appears to have been internally rotated at the ankle. It involves four major deformities, i.e. equinus deformity (shortening of the archiles tendon), heel varus (internal rotation at the ankle), medial cavus (a high medial arch due to pronation of the forefoot in relation to the hind foot), forefoot adduction (medial deviation of the forefoot) (Ponseti, 1997).

Developmental Milestone - The Medical Encyclopedia (2016) defines developmental milestones as behaviours or physical skills seen in infants and children as they grow and develop; rolling over, crawling, walking and talking are all considered as milestones.

\section{METHODOLOGY}

In this hospital-based prospective study, 48 children with idiopathic clubfoot were recruited from the Ponseti clubfoot clinic of the Lagos State University Teaching Hospital. Those included started treatment from the second week after birth, were full term and had no other orthopaedic conditions or previous clubfoot treatment. They were required to be compliant with the wearing of the foot abduction brace as prescribed. An eight-item questionnaire for determination of the ages of developmental milestones in children as used in the study by Sala et al (2013) was adapted and validated with Pearson correlation coefficient of 0.73 . The properties measured in the questionnaire are (Roll prone to supine, Roll supine to prone, Sit without support, Belly crawl, Crawl, Pull to stand, Stand unsupported, Walk unsupported). The research protocol was explained to the parents of the children enrolled. The questionnaire, containing both open- and close-ended questions, was given to the parents to fill. Parents were interviewed repeatedly to obtain the ages at which their children attained the eight developmental milestones. Sociodemographic data and ages at each milestone achievement were recorded and expressed as mean and standard deviation. An independent t-test was used to compare the mean between the ages at attainment of developmental milestone and normative data. The SPSS version 17 was used in the data analysis.

\section{RESULTS}

Forty-eight children participated in the study. Their ages ranged from 14 to 24 months with a mean of $16.44 \pm 10.31$ months. 38 were male and 10 were female giving a malefemale ratio of 3.8 to 1 (fig. 1).

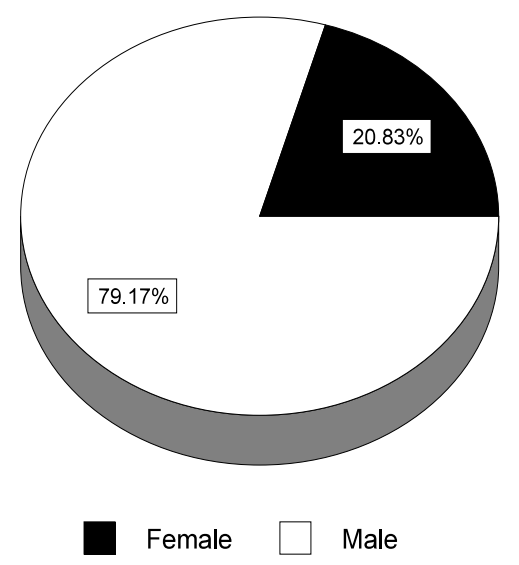

The mean age of milestone achievement revealed that the subjects achieved roll prone to supine at a mean age of 3.2 months, roll supine to prone at a mean age of 3.46 months, sit without support at a mean age of 5.46 months, 
belly crawl at a mean age of 6.98 months, crawl at a mean age of 7.73 months, pull to stand at a mean age of 9.96 months, stand unsupported at a mean age of 11.24 months, walk with support at a mean age of 12.7 months, walk unsupported at a mean age of 15.9 months (table 1 ).

Table1. Mean Age of Subjects at Milestone Achievement

\begin{tabular}{lll}
\hline Milestones & Mean & Standard Deviation \\
\hline Roll prone to supine & 3.27 & 0.64 \\
Roll supine to prone & 3.46 & 0.74 \\
Sit without support & 5.46 & 1.03 \\
Belly crawl & 6.98 & 1.10 \\
Crawl & 7.73 & 2.23 \\
Pull to Stand & 9.96 & 2.20 \\
Stand Unsupported & 11.24 & 1.97 \\
Walk with Support & 12.79 & 2.54 \\
Walk Unsupported & 15.90 & 6.84 \\
\hline
\end{tabular}

Compared with normative data only one of the mean ages of achievement out of the 8 milestones was significantly greater $(\mathrm{P}<0.05)$ for the children studied. Pre-ambulatory and crawling milestones showed no significant difference, but independent walking was achieved up to 2 months later.

\section{DISCUSSION}

Ponseti first described this method of treatment for clubfoot in 1963, but it took about 30 years before the orthopaedic community worldwide was able to catch up with him. However, factors such as the developmental milestones of the children treated were not considered in his study. In the present study, the children presented at an earlier age and an average of 4 to 6 casts with a mean of 4.6 were applied. Parents were interviewed repeatedly to obtain the ages when their children attained the developmental milestones. The results show that pre-ambulatory and crawling milestones were in consonance with normative data, but upright ambulation was achieved up to 2 months later. The results were different from that of a previous study done by Sala et al (2013) in which pre-ambulatory milestones were achieved between 0.7 and 1.5 months later and independent ambulation achieved up to 2.2 months later.

\section{CONCLUSION}

From the outcome of this study, it can be concluded that no significant delays in developmental milestones were found in children with idiopathic club foot treated with the Ponseti method except for upright ambulation which had delays of at most 2 months.

\section{References}

Adewole O.A., Giwa S.O., Kayode M.O., Shoga M.O., Balogun R.A. 2009. Congenital club foot in a teaching hospital in Lagos, Nigeria. African Journal of Medicine and Medical Sciences 38: 203-206.

Adewole O.A., Williams O.M., Kayode M.O., Shoga M.O., Giwa S.O. 2014. Early experience with Ponseti clubfoot management in Lagos, Nigeria. East and Central African Journal of Surgery 19(2): 71-76.

Dahaung Z., Jianlin L., Li Z., Zhenkai W. 2014. Relapse of clubfoot after treatment with Ponseti method and the function of clubfoot abduction orthosis. Journal of Clinical Orthopaedic Surgery 6(3): 245-552.

Dobbs M.B., Gurnett C.A. 2009. Update on clubfoot: Aetiology and treatment. Clinical Orthopaedics and Related Research 467: 1146-1153.

Morcuende J.A., Dolan L., Dietz F., Ponseti I.V. 2004. Radical reduction in the rate of extensive corrective surgery for clubfoot using Ponseti method. Journal of Paediatrics 113: 276 -380 .

Omololu B., Ogunlade S.O., Alonge T.O. 2005. Pattern of orthopaedic malformations in African teaching hospital. West African Journal of Medicine 24(2): 92-95.

Ponseti I.V. 1997. Common errors in the treatment of congenital clubfoot. International Journal of Orthopaedics 20(6): 699 700 .

Ponseti I.V., Smoley E.N. 1963. Congenital clubfoot: The results of treatment. Journal of Bone Joint Surgery 45(2): 2261-2270.

Ponseti IV 2000. Clubfoot management [Editorial]. Journal of Paediatric Orthopaedics 20(6): 699-700.

Sala D.A., Chu A., Lehman W.B., Van B., Harold J.P. 2013. Achievement of gross motor milestones in children with idiopathic clubfoot treated with the Ponseti method. Journal of Paediatrics Orthopaedics 33(1): 55-58.

Staheli, L. 2009. Clubfoot: Ponseti Management $3^{\text {rd }}$ edition, Global Help Publications, 04-27. 